\title{
ОЦЕНКА ИНВЕСТИЦИОННЫХ ПРОЕКТОВ В НЕФТЕПЕРЕРАБОТКЕ: МЕТОДИКА И МАТЕМАТИЧЕСКОЕ ОБЕСПЕЧЕНИЕ
}

\section{EVALUATION OF INVESTMENT PROJECTS IN OIL REFINING: METHODOLOGY AND MATHEMATICAL SUPPORT}

A. Sizikov

Summary. The features of the application of guidelines for assessing the effectiveness of investment projects in oil refining are considered. The software for predicting changes in technical and economic indicators in the process of phased reconstruction of production is proposed. An example of the use of the proposed tools for the analysis and comparison of investment projects of an existing enterprise is presented.

Keywords: oil refining; investments; software; evaluation of investment projects.

\author{
Сизиков Александр Павлович \\ К.э.н., дочент, Самарский государственный \\ экономический университет \\ apsizikov@mail.ru
}

Аннотация. Рассмотрены особенности применения методических рекомендаций по оценке эффективности инвестиционных проектов в нефтепереработке. Предложено математическое обеспечение для прогнозирования изменения технико-экономических показателей в процессе поэтапной реконструкции производства. Представлен пример использования предлагаемого инструментария для анализа и сравнения инвестиционных проектов действующего предприятия.

Ключевые слова: нефтепереработка; инвестиции; математическое обеспечение; оценивание инвестиционных проектов.

Для определения того, как изменения в параметрах технологического оборудования влияют на интенсивности и качественные характеристики материальных потоков, затраты топлива, электроэнергии и других ингредиентов и, в конечном счете, на экономические показатели, предлагается комплексная модель нефтеперерабатывающего производства [4].

Нефтеперерабатывающее производство можно представить в виде сетевого направленного графа. Множество вершин (узлов) этого графа $K=U \cup S$, где $U-$ множество установок, $S$ - множество смесевых пулов. Множество дуг отражает потоки нефтепродуктов. Пусть I - множество нефтепродуктов. Каждый продукт может быть представлен одним или несколькими потоками. Если $J_{i}-$ множество потоков, представляющих продукт $i \in I$, тогда множество всех потоков есть $J=\cup_{i \in I} J_{i}$.

Уравнения материального баланса:

$$
\sum_{j \in J_{i}^{+}} x_{j}-\sum_{j \in J_{i}^{-}} x_{j}=0, i \in I,
$$

где $x_{j}-$ интенсивность $j$-го потока; $J_{i}^{+}\left(J_{i}^{-}\right)$- множество потоков пополняющих (расходующих) $i$-й продукт.

Ограничения по поставкам сырья и полуфабрикатов со стороны:

$$
P_{i}^{-} \leq \sum_{j \in J_{i}^{+}} x_{j} \leq P_{i}^{+}, i \in I^{+},
$$


где $I^{+}$- множество продуктов, поступающих со стороны; $P_{i}^{-}\left(P_{i}^{+}\right)$- пределы поставки.

Требования по отгрузке товарной продукции:

$$
V_{i} \leq \sum_{j \in J_{i}^{-}} x_{j} \leq V_{i}^{+}, i \in I^{-},
$$

где $I^{-}$- множество товарных продуктов; $V_{i}^{-}\left(V_{i}^{+}\right)-$ пределы отгрузки товарного продукта.

Материальные балансы узлов:

$$
\sum_{j \in J_{k}^{+}} x_{j}-\sum_{j \in J_{k}^{-}} x_{j}=0, k \in K,
$$

где $J_{k}^{+}\left(J_{k}^{-}\right)$- множество входных (выходных) потоков $k$-го узла.

Ограничения по загрузке узлов:

$$
L_{k}^{-} \leq \sum_{j \in J_{k}^{+}} x_{j} \leq L_{k}^{+}, k \in K,
$$

где $L_{k}, L_{k}^{+}$- пределы загрузки (потенциал) $k$-го узла.

Установка, как узел сетевой модели, представляется конечным множеством $R_{k}$ технологических режимов. Соответственно вводятся переменные $\left\{x_{j r}, r \in R_{k}\right\}$ , где $x_{j r}$ - интенсивность $j$-го потока для режима $r$. Для каждого технологического режима $r \in R_{k}$ могут быть заданы ограничения в виде допустимых интервалов варьирования входных потоков $-\left[\delta_{j r}^{-}, \delta_{j r}^{+}\right], j \in J_{k}^{+}$, где $\delta_{j r}^{-}, \delta_{j r}^{+}-$соответственно нижний и верхний пределы доли потока в общей загрузке установки. Выходы установки определяются так:

$$
x_{i r}=\sum_{j \in J_{k}^{+}} a_{i j r} x_{j r}, i \in J_{k}^{-},
$$

где $a-$ коэффициенты линейного преобразования.

Для связи вновь введенных переменных с переменными основной группы вводятся балансовые уравнения

$$
x_{j}-\sum_{r \in R_{k}} x_{j r}=0, j \in J_{k}^{+} \cup J_{k}^{-},
$$

и для каждого $r \in R_{k}$ вводится блок

$$
\left\{\begin{array}{l}
\sum_{j \in J_{k}^{+}} \alpha_{i j r}^{-} x_{j r} \geq 0, i \in J_{k}^{+}, \\
\sum_{j \in J_{k}^{+}} \alpha_{i j r}^{+} x_{j r} \leq 0, i \in J_{k}^{+}, \\
\sum_{j \in J_{k}^{+}} a_{i j r} x_{j r}-x_{i r}=0, i \in J_{k}^{-}, \\
X_{j r} \geq 0, j \in J_{k}^{+} \cup J_{k}^{-},
\end{array}\right.
$$

где $\alpha_{i j r}^{-}=\left\{\begin{array}{c}1-\delta_{i r}^{-}, i=j, \\ -\delta_{i r}^{-}, i \neq j,\end{array}, \alpha_{i j r}^{+}=\left\{\begin{array}{c}1-\delta_{i r}^{+}, i=j, \\ -\delta_{i r}^{+}, i \neq j\end{array}\right.\right.$
Смесевой пул можно рассматривать как узел с несколькими входящими потоками (компонентами смешения) и одним исходящим - продуктом смешения. Пропорции компонентов смешения определяются требованиями по качеству товарных смесей, которые можно в первом приближении представить следующим образом:

$$
\begin{aligned}
& I\left(p_{q}^{-}\right) \sum_{j \in J_{k}^{+}} \frac{x_{j}}{p_{o j}} \leq \sum_{j \in J_{k}^{+}} I\left(p_{q j}\right) \frac{x_{j}}{p_{o j}} \leq I\left(p_{q}^{+}\right) \sum_{j \in J_{k}^{+}} \frac{x_{j}}{p_{o j}} \\
& q \in Q_{k}
\end{aligned}
$$

где $Q_{k}$ - множество параметров качества для $k$-го продукта смешения; $p_{q j}-$ значение $q$-го параметра $j$-го компонента; $p_{q}^{-}, p_{q}^{+}$- нижняя и верхняя границы параметра для продукта; $p_{o j}$ - базовый параметр для пересчета значения величины $p_{q j}$ на единицу массы; $I(p)$ - индекс параметра (индексные преобразования выполняются для некоторых параметров для обеспечения аддитивности модели смешения по этим параметрам).

Представляя ограничения (8) в виде, при котором все переменные находятся в левой части, для каждого $k \in S$ запишем:

$$
\begin{aligned}
& \left\{\begin{array}{l}
\sum_{j \in J_{k}^{+}} \alpha_{i j}^{-} x_{j} \geq 0, i \in J_{k}^{+}, \\
\sum_{j \in J_{k}^{+}} \alpha_{i j}^{+} x_{j} \leq 0, i \in J_{k}^{+}, \\
\sum_{j \in J_{k}^{+}} \beta_{q j}^{-} x_{j} \geq 0, q \in Q_{k}, \\
\sum_{j \in J_{k}^{+}} \beta_{q j}^{+} x_{j} \leq 0, q \in Q_{k},
\end{array}\right. \\
& \text { где } \alpha_{i j}^{-}=\left\{\begin{array}{c}
1-\delta_{k j}^{-}, i=j, \\
-\delta_{k j}^{-}, i \neq j,
\end{array} \alpha_{i j}^{+}=\left\{\begin{array}{c}
1-\delta_{k j}^{+}, i=j, \\
-\delta_{k j}^{+}, i \neq j,
\end{array}\right.\right. \\
& \beta_{q j}^{-}=\frac{I\left(p_{q j}\right)-I\left(p_{q}^{-}\right)}{p_{o q}}, \beta_{q j}^{+}=\frac{I\left(p_{q j}\right)-I\left(p_{q}^{+}\right)}{p_{o q}}
\end{aligned}
$$

По переменным модели можно рассчитать экономические показатели. Прибыль определяется следующим образом:

$$
\Pi=D-(V+C)=(D-V)-C=P-C,
$$

где $D-$ стоимость товарной продукции; $V$ - переменные затраты; $C$ - условно-постоянные затраты; $P$ покрытие (маржинальный доход).

$$
P=\sum_{i \in I^{-}} c_{i} \sum_{j \in J_{i}^{-}} x_{j}-\left(\sum_{i \in I^{+}} c_{i} \sum_{j \in J_{i}^{+}} x_{j}+\sum_{k \in K} \varsigma_{k} \sum_{j \in J_{k}^{+}} x_{j}\right)
$$


Таблица 1. Инвестиции в проекты

\begin{tabular}{|c|c|c|c|c|}
\hline \multirow{2}{*}{ Статьи затрат, млн. руб. } & \multicolumn{4}{|c|}{ Варианты проектов } \\
\hline & 1 & 2 & 3 & 4 \\
\hline Монтажные работы & 6429,4 & 6517,7 & 7769,7 & 16634,2 \\
\hline Стоимость оборудования & 6497,2 & 8688,8 & 6887,5 & 21106,4 \\
\hline Прочие расходы & 2084,8 & 3311,9 & 2596,6 & 8473,5 \\
\hline Итого & 14914,4 & 18509,7 & 17253,8 & 45827,1 \\
\hline Этапы инвестиций & \multicolumn{4}{|c|}{ Капитальные вложения } \\
\hline 0 & 147,90 & 186,3 & 173,6 & 457,9 \\
\hline 1 & 597,45 & 744,5 & 687,9 & 1833,8 \\
\hline 2 & \begin{tabular}{|l|}
1487,34 \\
\end{tabular} & 1855,7 & 1725,6 & 4584,8 \\
\hline 3 & 3728,32 & 4626,4 & 4315,4 & 11456,6 \\
\hline 4 & 4771,54 & 5934,6 & 5527,6 & 14754,7 \\
\hline 5 & 4173,54 & 5187,8 & 4837,8 & 12837,9 \\
\hline
\end{tabular}

где $c_{i}$ - цена $i$-го продукта (для промежуточных продуктов эта величина равна нулю); $\varsigma_{k}$ - удельные затраты, связанные с загрузкой $k$-го узла (стоимость топлива, электроэнергии, расходных ингредиентов).

Кроме прибыли, по интенсивностям потоков рассчитываются показатели, специфические для нефтепереработки. К их числу относятся, например, выход светлых нефтепродуктов и глубина переработки нефти.

Технико-экономические показатели приводятся к сопоставимому безразмерному виду, нормализуются относительно каких-либо целевых или номинальных значений. Затем из них с учетом весовых коэффициентов формируется свертка (выпуклая линейная комбинация), которая берется в качестве критерия [5].

В обобщенной векторной форме задача (1) - (11) выглядит следующим образом:

$$
\begin{aligned}
& (\mathbf{p}, \boldsymbol{\delta}) \rightarrow \min , \\
& \left\{\begin{array}{l}
\boldsymbol{\delta}=(\mathbf{E g})^{-1}(\mathbf{A x}-\mathbf{g}), \\
\mathbf{b}^{-} \leq \mathbf{B x} \leq \mathbf{b}^{+}, \\
\mathbf{x} \geq \mathbf{0},
\end{array}\right.
\end{aligned}
$$

где $x$ - вектор интенсивностей материальных потоков; $g$ - вектор целевых показателей; $A$ - матрица линейного преобразования вектора переменных в вектор показателей; $B$ - матрица производственно-технологических параметров; $b^{-}, b^{+}$- векторы производственно-технологических ограничений; $\ddot{a}-$ вектор относительных отклонений расчетных значений показателей от заданных; $p$ - вектор приоритетов показателей; $E-$ единичная матрица.

С помощью модели (12) можно определить связь между технологическими параметрами производства и его технико-экономическими показателями. На базе этой модели разработана компьютерная программа СМОННП [6]. Программа применяется следующим образом. На начальном этапе вводятся параметры действующего предприятия. Берутся номинальные загрузки установок, коэффициенты отбора нефтепродуктов, типовые спецификации товарной продукции, нормативные энергетические затраты. Показатели вводимых процессов берутся из технической документации фирм-производителей. Далее для каждого проекта рассчитывается оптимальная траектория развития предприятия по годам, учитывающая ввод в эксплуатацию новых установок или реконструкцию уже существующих [7].

Ниже представлены результаты использования программы в рамках стандартной методики для исследования различных вариантов реконструкции нефтеперерабатывающего предприятия. Варианты реконструкции состоят в том, что к существующему составу производственного оборудования добавляются различные установки. Рассматривались следующие варианты:

1. Вакуумная трубчатая установка мощностью 1,8 млн.т/год.

2. Установка деасфальтизации (1,6 млн.т/год), установка флексикокинга (1,0 млн.т/год).

3. Установка деасфальтизации (1,6 млн.т/год) и установка замедленного коксования (1,0 млн.т/год).

4. Установка гидрокрекинга (1,7 млн.т/год), установка производства водорода (65тыс.т/год) и установка производства серы мощностью (30 тыс.т/ год).

Вложения в проекты по всем вариантам, разбитые по годам, показаны в табл. 1.

Для действующего производства и вариантов реконструкции рассчитаны материальные балансы - развернутый и сводный. По ним рассчитываются загрузки установок и требуемые ресурсы. А также производственные 
Таблица 2. Результаты реализации инвестиционных проектов

\begin{tabular}{|c|c|c|c|c|c|}
\hline \multirow{2}{*}{ Статьи затрат и результатов, млн.руб. } & \multicolumn{5}{|c|}{ Проекты } \\
\hline & База & 1 & 2 & 3 & 4 \\
\hline Сырье & 28885 & 29088 & 29166 & 29074 & 30753 \\
\hline Материалы & 12709 & 14670 & 14180 & 14129 & 12958 \\
\hline Товарная продукция & 90723 & 98629 & 96419 & 97178 & 109626 \\
\hline Общий доход & 48949 & 54813 & 53274 & 53985 & 63941 \\
\hline Доход к базе & 0 & 6143 & 4426 & 5157 & 17192 \\
\hline
\end{tabular}

Таблица 3. Динамика показателей 1-го проекта

\begin{tabular}{|l|l|l|l|l|l|l|}
\hline Год & $\mathbf{2}$ & $\mathbf{3}$ & $\mathbf{4}$ & $\mathbf{5}$ & $\mathbf{6}$ \\
\hline 0 & $-147,81$ & & 1 & $-147,81$ & $-147,81$ \\
\hline 1 & $-595,42$ & & 0,834 & $-498,10$ & $-645,88$ \\
\hline 2 & $-1488,26$ & & 0,693 & $-1035,27$ & & $-1679,89$ \\
\hline 3 & $-3728,22$ & & 0,581 & $-2157,95$ & & $-3837,13$ \\
\hline 4 & $-4771,62$ & & 0,483 & $-2301,04$ & & $-6137,61$ \\
\hline 5 & $-4172,24$ & & 0,403 & $-1677,03$ & & $-7814,08$ \\
\hline 6 & & 6043,00 & 0,336 & & 2027,16 & $-5794,03$ \\
\hline 7 & & 6043,00 & 0,278 & & 1687,32 & $-4114,19$ \\
\hline 8 & & $0,43,00$ & 0,232 & & 1405,17 & $-2696,52$ \\
\hline 9 & & 6043,00 & 0,195 & & 1171,87 & $-1531,24$ \\
\hline 10 & & 6043,00 & 0,163 & & 975,83 & $-552,24$ \\
\hline 11 & & 6043,00 & 0,136 & & 67,29 & 263,17 \\
\hline 12 & & 6043,00 & 0,114 & & 565,03 & 939,03 \\
\hline 13 & & 6043,00 & 0,094 & & 471,18 & 1513,04 \\
\hline 14 & & 6043,00 & 0,075 & & 391,97 & 1970,14 \\
\hline 15 & & 6043,00 & 0,067 & & 10178,81 & 2367,18 \\
\hline
\end{tabular}

Таблица 4. Показатели проектов

\begin{tabular}{|l|l|l|l|l|}
\multirow{2}{*}{ Показатели проектов } & \multicolumn{4}{l|}{ Варианты проектов } \\
\cline { 2 - 6 } & $\mathbf{1}$ & $\mathbf{2}$ & $\mathbf{3}$ & $\mathbf{4}$ \\
\hline NPV & 2367,18 & $-2235,96$ & $-369,07$ & 4781,76 \\
\hline$P$ & 1,32 & 0,75 & 1,04 & 1,30 \\
\hline$I R R$ & 0,28 & 0,17 & 0,25 & 0,22 \\
\hline
\end{tabular}

результаты - стоимость товарной продукции, прибыль и другие. Финансовые результаты рассматриваемых проектов представлены в табл. 2.

Пример расчета 1-го варианта приведен в табл. 3, где 1 - вложения в проект по годам, 2 - отдача по годам, 3 - дисконтирующий коэффициент, 4 - дисконтированные затраты, 5 - дисконтированные результаты, 6 $N P V$ нарастающим итогом.
Итоговые показатели проекта: $N P V=2367,18$; $P=1,32 ; I R R \approx 0,28$.

Описанным способом рассчитываются показатели других инвестиционных вариантов. Результаты по всем вариантам представлены в табл. 4. Как видим, наилучшие показатели у проектов 1 и 4. Наибольшее значение $N P V$ у проекта 4, но он несколько уступает первому варианту по остальным показателям. 
Резюме. Необходимым этапом процедуры оценивания инвестиционных проектов в нефтепереработке является расчет элементов потока затрат и результатов. Такой расчет невозможно сделать без использования инструментария, позволяющего определить, как те или иные изменения производственно-технологических параметров отражаются на технико-экономических показателях. В качестве такого инструментария предложена авторская программа СМОННП.

Программа использовалась для анализа вариантов модернизации ряда предприятий: Ангарской нефтехимической компании, Куйбышевского НПЗ, Новокуйбышевского НПЗ, Сызранского НПЗ, Новокуйбышевского завода масел и присадок, Орского НПЗ и других.

\section{ЛИТЕРАТУРА}

1. Кутузова М. Н. Модернизация НПЗ и мировые тренды в нефтепереработке. Neftegaz.ru. 2018, № 4(76)

2. Методические рекомендации по оценке эффективности инвестиционных проектов URL: http://www.consultant.ru/document/cons_doc_LAW_28224/

3. Андреев, А. Ф. Оценка эффективности и планирование проектных решений в нефтегазовой промышленности. — М.: Нефть и газ. 1997. - 276 с

4. Сизиков, А. П. Оптимизация нефтеперерабатывающего производства как сложной системы. Вестник Самарского государственного технического университета. 2010, № 7(28).

5. Livshits M. Yu., Sizikov A. P. Multi-Criteria Optimization of Refinery Thermophysical Basis of Energy Technologies 2015 (01035) EPJ Web Conf. 110: Les Ulis Cedex France: Web of Conferences. 2016. DOl: http: dx.DOl.org /10.1051/ epjconf / 201611001035

6. Сизиков, А. П. Программный продукт СМОННП (система оптимизации нефтеперерабатывающих и нефтехимических производств). Управление большими системами/ Сборник трудов. Выпуск 24: М.: ИПУ РАН, 2009- С. 298-326

7. Копасева, Л.А., Сизиков, А. П. Обоснование инвестиционных проектов в нефтепереработке: программный продукт СМоннП. // Проблемы развития предприятий: теория и практика. Материалы 6-й Международной научно-практической конференции. Самара Октябрь 2007.— C. 383-386

(с) Сизиков Александр Павлович ( apsizikov@mail.ru).

Журнал «Современная наука: актуальные проблемы теории и практики»

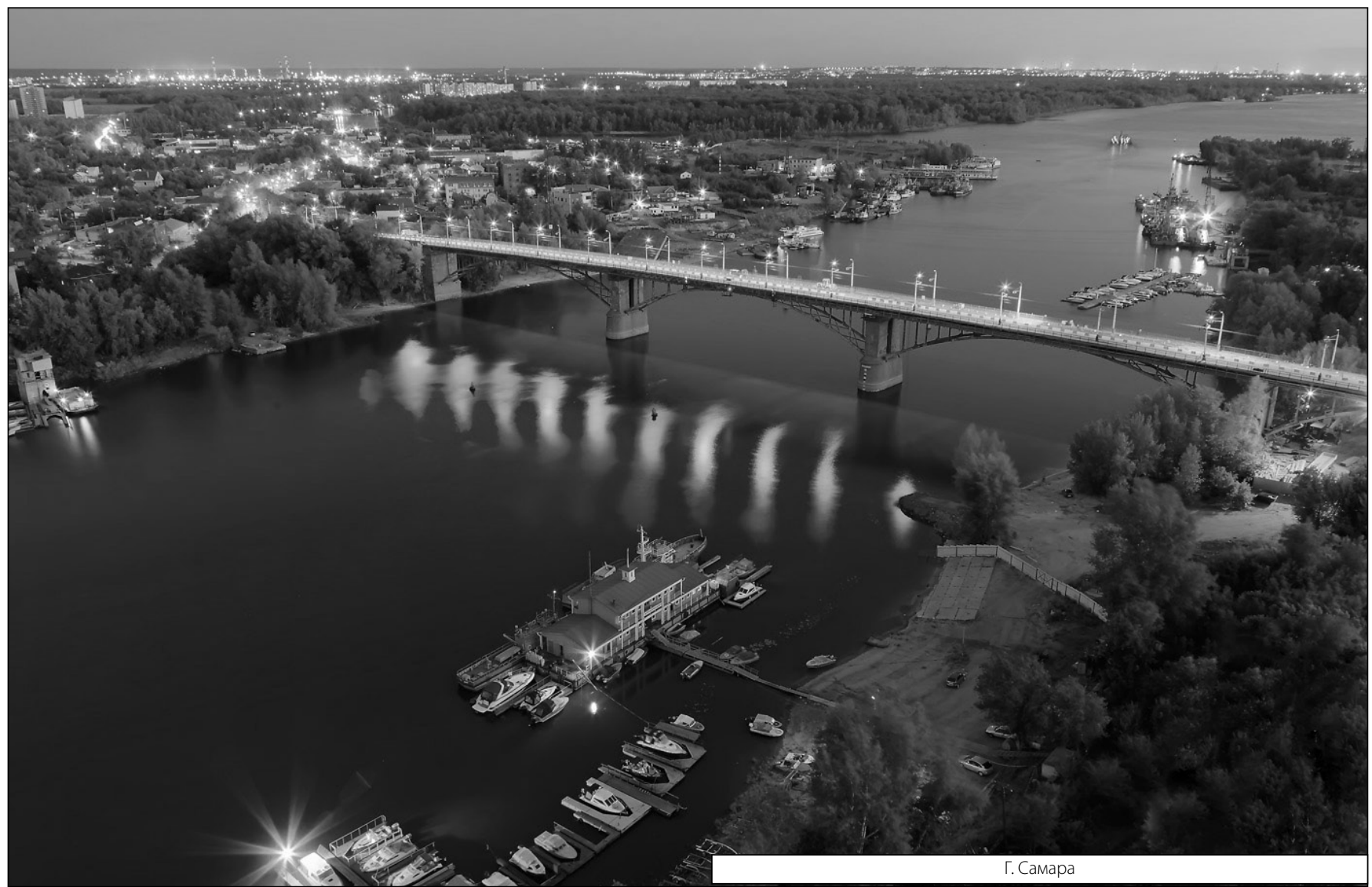

